\title{
Differential microRNA expression profiles in HCT116 colorectal cancer cell lines located in the lung and colon
}

\author{
XIAO-HONG WANG ${ }^{1}$, XIN-MIN YU ${ }^{1}$, HONG JIANG ${ }^{2}$ and CONG LUO ${ }^{1}$ \\ ${ }^{1}$ Department of Chemotherapy, Zhejiang Cancer Hospital, Hangzhou, Zhejiang 310022; \\ ${ }^{2}$ Department of Thoracic Surgery, Hangzhou People's First Hospital, Hangzhou, Zhejiang 310006, P.R. China
}

Received February 9, 2014; Accepted November 4, 2014

DOI: $10.3892 / \mathrm{mmr} .2014 .3010$

\begin{abstract}
Colorectal cancer (CRC) is one of the most common types of cancer worldwide. The majority of mortalities caused by colorectal cancer are due to metastatic disease. As numerous CRC patients experience metastasis to the liver or lung and fail to respond to curative therapies, intensive research efforts have sought to identify the molecular changes or regulatory mechanisms underlying CRC metastasis. In the present study, a stable CRC cell line, HCT16, overexpressing firefly luciferase was constructed and an in vivo metastasis model was established via intravenous injection of this cell line. Using an imaging system, tumor tissue located in the lung and colon was separated and cells were prepared. The microRNA (miRNA) expression profiles of these lung homing or colon homing cells were assessed and compared. A total of 38 differentially expressed miRNAs were selected and confirmed our previous results; several of these have been reported to be involved in the regulation of cancer progression. However, the remaining miRNAs require further investigation. The present profiling may be the first step toward delineating the differential expression of miRNAs in the CRC cells located in the colon and the lung, enabling the elucidation of the regulation associated with miRNAs in colorectal lung metastases. These miRNAs require further validation and functional analysis to evaluate whether they are important in the pathogenesis of colorectal lung metastases or are adopted as markers to predict colorectal metastasis.
\end{abstract}

\section{Introduction}

Colorectal cancer (CRC) is one of the most common types of cancer worldwide, accounting for $\sim 9 \%$ of all cancer mortality in 2013 (1). Metastatic disease develops in $>50 \%$ of patients,

Correspondence to: Dr Hong Jiang, Department of Thoracic Surgery, Hangzhou People's First Hospital, 261 Huansha Road, Hangzhou, Zhejiang 310006, P.R. China

E-mail: hh110120@126.com

Key words: microRNA expression profiles, colorectal cancer, HCT116, metastasis exhibiting an ultimately fatal prognosis, with 5-year survival rates of $10 \%$ (2). Although treatment with chemotherapy and radiation therapy has improved and become more effective for locally advanced mid to low rectal cancers and metastatic colon lesions over the past three decades (3), to date, no major advance has been observed in 5-year survival rates and the number of patients cured from metastatic CRC remains low.

Metastasis is a complex process involving multiple steps. Tumor cells are required to achieve the two rate-limiting steps of invasion and distant colony formation. Metastatic spread to the liver and lungs occurs most frequently in colorectal cancer metastases in humans (4). Numerous CRC patients experience metastasis to the liver or lung and fail to respond to curative therapies, thus significant research efforts have focused on identifying the molecular changes underlying CRC metastasis (5). Several candidate biomarkers have been reported, including ST6 galactose I and $\beta 1$ integrin (6,7). However, the mechanism of distant metastasis in CRC remains to be elucidated.

MicroRNAs (miRNAs) are a group of small, endogenous, non-protein-coding RNA molecules that negatively regulate gene expression $(8,9)$. In previous decades, miRNAs were found to be important in multiple biological processes and the metabolic regulation of cancer (10). In addition, multiple studies have indicated the importance of miRNAs in managing the efficiency of chemotherapy in several types of human cancer $(11,12)$. In the present study, the CRC cell line HCT116 was infected with a recombinant lentivirus carrying the firefly luciferase gene and a stable cell line was obtained via puromycin selection (HCT116/Luc). This cell line was selected as an in vivo model of metastasis (13). The present study aimed to investigate the differences of miRNA expression profiles between the HCT116/Luc located in the colon and lung, respectively.

\section{Materials and methods}

Preparation of the lentivirus. All cell culture reagents were purchased from Invitrogen Life Technologies (Carlsbad, CA, USA). The firefly luciferase gene was inserted into the lentivirus vector Ubi-MCS-IRES-Puromycin to obtain plasmid Ubi-Luc-IRES-Puromycin. HEK293T cells, obtained from the Type Culture Collection of the Chinese Academy of Sciences, (Shanghai, China), were plated at a density of $1 \times 10^{6}$ cells per $100 \mathrm{~mm}$ dish $24 \mathrm{~h}$ prior to transfection. The 
cells were transfected with Ubi-Luc-IRES-Puromycin along with packaging vectors, pMD2G and psPAX2 according to the manufacturer's instructions for Lipofectamine 2000. The transfection medium was removed the following day and replaced with Dulbecco's modified Eagle's medium supplemented with $10 \%$ fetal bovine serum (FBS). The supernatants from these cells were collected $72 \mathrm{~h}$ after recovery from transfection. The supernatants were filtered using a $0.45 \mu \mathrm{M}$ syringe filter, combined with $4 \mu \mathrm{g} / \mathrm{ml}$ of polybrene and added to the cultured human CRC cell line HCT116.

Cell culture and transfection. The HCT116 cell line was obtained from the cell bank of the Chinese Academy of Sciences (Beijing, China). Cells were cultured in RPMI-1640 supplemented with 10\% FBS (complete media). They were infected with the recombinant lentivirus carrying the firefly luciferase gene and a stable cell line (HCT116-Luc) was obtained via puromycin selection. The expression of luciferase was confirmed by a luciferase assay system kit purchased from Promega Corporation (Madison, WI, USA) according to the manufacturer's instructions.

Cell line subselection using an in vivo model of metastasis. HCT116-Luc cells were collected and resuspended in phosphate-buffered saline at a concentration of $4 \times 10^{7}$ cells $/ \mathrm{ml}$. Balb/c null mice (Shanghai SLAC Laboratory Animal Co., Ltd., Shanghai, China) were inoculated with $0.1 \mathrm{ml}$ cell suspension through a tail vein. On day 28 , tumor metastasis in each mouse was monitored using the IVIS Lumina II in vivo imaging system (Xenogen, Alameda, CA, USA). Tumor tissues were isolated from mice that had significant lung and colon metastases. A single cell suspension was prepared using a trypsin digestion method. Briefly, tumor tissues were cut into small sections and digested with $0.25 \%$ trypsin at $4{ }^{\circ} \mathrm{C}$ overnight, followed by incubation at $37^{\circ} \mathrm{C}$ for $30 \mathrm{~min}$. Following removal of any tissue residue by centrifugation at $300 \mathrm{x}$ g for $5 \mathrm{~min}$, cells were suspended with complete media supplemented with $2 \mu \mathrm{g} / \mathrm{ml}$ of puromycin. Following three rounds of subselection, HCT116/ Luc-P (cells isolated from the lung) and HCT116/Luc-I (cells isolated from the colon) were obtained. The present study was approved by the ethics committee of Shanghai University of Traditional Chinese Medicine (Shanghai, China)

RNA isolation. Cells were seeded at a density of 600,000 cells/well in a flat-bottom 6-well plate in $2 \mathrm{ml}$ of complete media. After $48 \mathrm{~h}$, cells were collected and total RNA was prepared using TRIzol (Invitrogen Life Technologies) according to the manufacturer's instructions with the exception that the precipitation was allowed to remain for $12 \mathrm{~h}$ at $-20^{\circ} \mathrm{C}$, which efficiently recovers all RNA species, including miRNAs. The RNA concentration was determined using a Nanodrop $^{\text {TM }}$ spectrophotometer (NanoDrop Technologies, Inc., Rockland, DE, USA). RNA integrity was assessed using a Tris-acetate-EDTA buffered agarose gel electrophoresis, analyzing integrity and association between the 28S rRNA, 18S rRNA and 5S rRNA bands.

Microarray miRNA profiling. The stacking-hybridized universal tag (SHUT) assay presented by Duan et al (14) is an efficient technique for high-throughput miRNA profiling using microarrays. Following the techniques described by Duan et al, a full-spectrum microarray was designed targeting each of the 2,017 mature human miRNAs listed in the Sanger miRBase version 19 (Wellcome Trust Sanger Institute, Hinxton, UK). Using the Agilent microarray (Agilent Technologies, Inc., Santa Clara, CA, USA), miRNA profiles of the HCT116/Luc-P and HCT116/Luc-I were obtained following the procedures as described by Zhang and Li (15).

Image scanning and data analysis. Following hybridization and washing, slides were scanned using a GenePix 4100A microarray scanner (Molecular Devices, Inc., Sunnyvale, CA, USA) at constant power and photomultiplier tube gain settings through a single-color channel (wavelength of $532 \mathrm{~nm}$ ). The raw pixel intensities were extracted using the GenePix Pro 7.0 software (Molecular Devices, Inc.). Data processing, including filtration, background-correction, transformation and normalization as well as subsequent statistical analysis was performed using the methods provided by Duan et al (14). Finally, a heatmap was plotted to demonstrate the differential expression level of the mature miRNAs between HCT116/Luc-P and HCT116/Luc-I.

\section{Results}

Construction of the stable cell line HCT116/Luc. To obtain the stable cell line HCT116/Luc, the CRC cell line HCT116 was transfected with lentivirus-mediated luciferase, selected using puromycin and detected using a luciferase assay kit (cat no. E4030, Promega, Shanghai, China). As shown in Fig. 1, the luminescent signal of the selected cells were significantly higher than for the control HCT116 cells.

Selection of HCT116/Luc-P and HCT116/Luc-I using an in vivo model of metastasis. Four weeks after inoculation of the mice with HCT116/Luc, tumor metastasis in each mouse was monitored using the IVIS Lumina II in vivo imaging system (Caliper Life Sciences, Hopkinton, MA, USA) as shown in Fig. 2. Mice that exhibited significant lung and colon metastasis were selected and tumor tissues were isolated and cultured. Following three rounds of subselection, HCT116/ Luc-P and HCT116/Luc-I were obtained and identified using a luciferase assay system.

DifferentialmiRNA expression profilesbetween HCT116/Luc-P and HCT116/Luc-I. Based on the SHUT assay, the miRNA expression profiles in HCT116/Luc, HCT116/Luc-P and HCT116/Luc-I were obtained and the heat map of partial results is shown in Fig. 3. Of the 2,017 human miRNAs analyzed, 38 miRNAs were detected to be expressed differentially between cells of HCT116/Luc-I and HCT116/Luc-P (Table I; Fig. 3). Additionally, 26 of the 38 miRNAs were observed to be expressed at a level $>2$-fold higher in HCT116/Luc-I than in HCT116/Luc-P, while the remaining 12 miRNAs demonstrated significantly lower expression.

\section{Discussion}

miRNAs are important in several types of human cancer. miRNAs have been demonstrated to be involved in the 


\section{Luciferase assay of HCT116/Luciferase cell line}

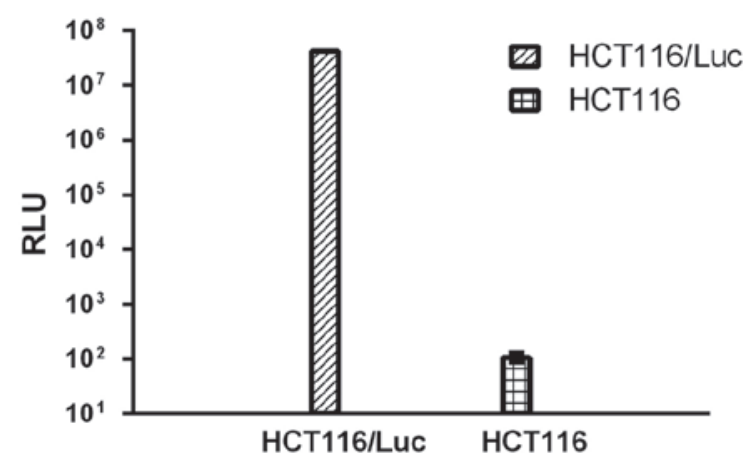

Figure 1. Luciferase assay of the stable cell line HCT116/Luc. The signal of the HCT116/Luc was over $1 \times 10^{5}$-fold greater than HCT116. RLU, relative light units.

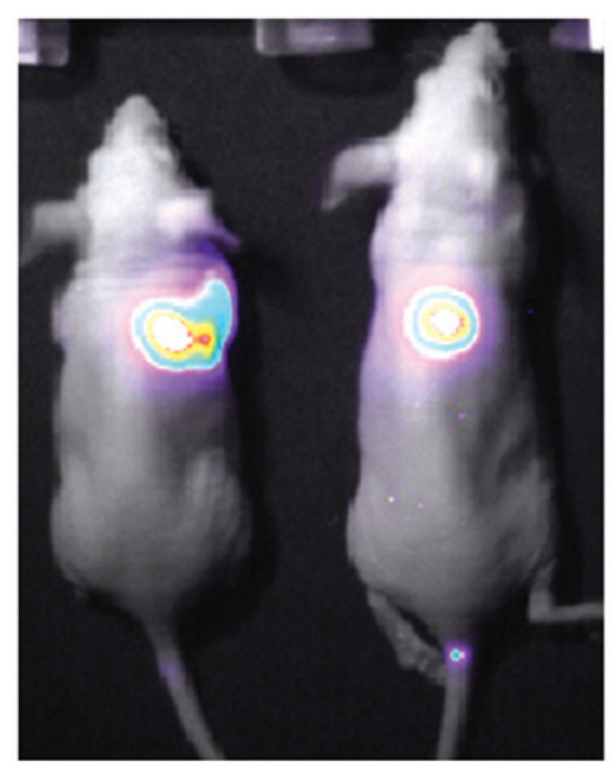

HCT116/Luc-P

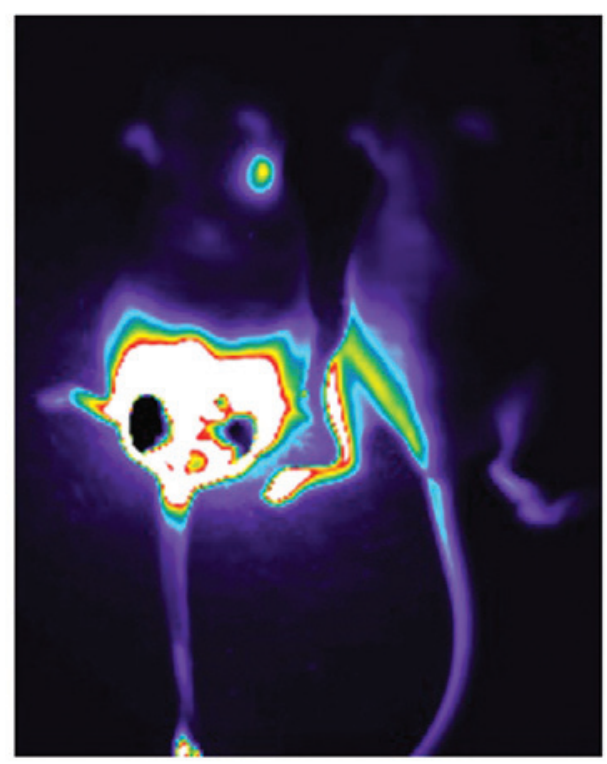

HCT116/Luc-I

Figure 2. In vivo imaging of mice inoculated with HCT116/Luc. regulation of expression of a number of cancer-associated genes $(8,16)$. Extensive research is currently focused on identifying miRNAs that may be important in cancer therapy and diagnosis. To date, more than 2,000 human miRNAs have been identified. These small RNAs impact many cellular processes and their deregulation is causative of many human cancers. In addition, many miRNAs were reported as predictive biomarkers and therapeutic targets in several cancers (17-20).

CRC is the third most common type of cancer in males and females (21). One of the hallmarks of CRC is the ability to invade adjacent structures and to metastasize to remote organs $(22,23)$. CRC is the second leading cause of cancer-associated mortality in the USA (24). The majority of mortalities caused by colorectal cancer are due to metastatic disease (4). As numerous CRC patients experience metastasis to the liver or lung and fail to respond to curative therapies, significant research efforts have aimed to identify the molecular changes or regulatory mechanisms underlying CRC metastasis (5). The present study, focusing on the differences of miRNA expression in HCT16 cell lines located in the lung and colon aimed to examine the potential miRNA targets associated with the regulation of colorectal lung metastases.

As shown in the results, the expression of 38 miRNAs was significantly different (all $\mathrm{P}<0.05$ ) in HCT116/Luc-P and HCT116/Luc-I. Notably, when comparing the miRNA expression profile of HCT116/Luc, 35 of the 38 miRNAs exhibited similar expression levels in HCT116/Luc and HCT116/ Luc-I while significantly different levels were observed in HCT116/Luc-P. This phenomenon may suggest that in the population of the CRC cell line HCT116, only a small number demonstrate metastastic characteristics.

A total of 26 miRNAs (shown in Table I) were revealed to be expressed at a level >2-fold greater in HCT116/Luc-I than in HCT116/Luc-P. Among these miRNAs, several have been reported to be downregulated in cancer cells or tissues in previous years (25-29). Hsa-miR-99a-5p was demonstrated to be downregulated $>2$-fold in squamous cell lung carcinoma tissues, compared with normal tissues (25). In the present study, the expression of Hsa-miR-99a-5p in HCT116/Luc-P was $>20$-fold lower than that in HCT116/Luc-I, which suggests that colorectal lung metastases cells may have partial lung carcinoma characteristics. Consistent with the results reported in the present study, several of these miRNAs have been previously reported to be tumor suppressors or downregulated in cancer cells. Hsa-miR-145-5p was reported to be downregulated in intracranial aneurysms (26). Knocking down hsa-miR-135b-5p in cell lines led to the depletion of the pediatric cancer stem cell fraction and impairment of sphere formation (27). In various breast cancer cell lines, the expression of hsa-miR-9-3p was low and synthetic-enhancer tumor suppressor characteristics were exhibited (28). Previously, Hsa-miR-7-5p expression was reported to be reduced in metastatic melanoma-derived cell lines compared with primary melanoma cells (29). This is consistent with the current results and possibly indicates that hsa-miR-7-5p may be important in the regulation of carcinoma cell line metastasis.

A total of 12 miRNAs (shown in Table I) were demonstrated to be overexpressed in HCT116/Luc-P. Among these miRNAs, hsa-miR-30a-5p has been previously reported to be overexpressed in glioma cell lines and glioma samples and its 
Table I. Comparison of miRNA expression profiles of HCT116 cells located in the lung and colon.

miRNA category Number miRNAs

Upregulated in 26

HCT116/Luc-I

26

Downregulated in

12

HCT116/Luc-I
hsa-miR-153, hsa-miR-99a-5p, hsa-miR-100-5p, hsa-miR-145-5p, hsa-miR-125b-5p, hsa-miR-4324, hsa-miR-7-5p, hsa-miR-203a, hsa-miR-31-5p, hsa-miR-191-5p, hsa-miR-222-3p, hsa-miR-9-3p, hsa-miR-378b, hsa-miR-151a-5p, hsa-miR-151b, hsa-miR-141-3p, hsa-miR-301a-3p, hsa-miR-135b-5p, hsa-miR-301b, hsa-miR-221-3p, hsa-miR-135a-5p, hsa-miR-151a-3p, hsa-miR-378i, hsa-miR-2392, hsa-miR-378c, hsa-miR-378a-3p

hsa-miR-10b-5p,hsa-miR-10a-5p,hsa-miR-30a-5p,hsa-miR-181b-5p, hsa-miR-3135b, hsa-miR-181a-5p, hsa-miR-22-3p, hsa-miR-224-5p, hsa-miR-4281, hsa-miR-708-5p, hsa-miR-192-5p, hsa-miR-155-5p
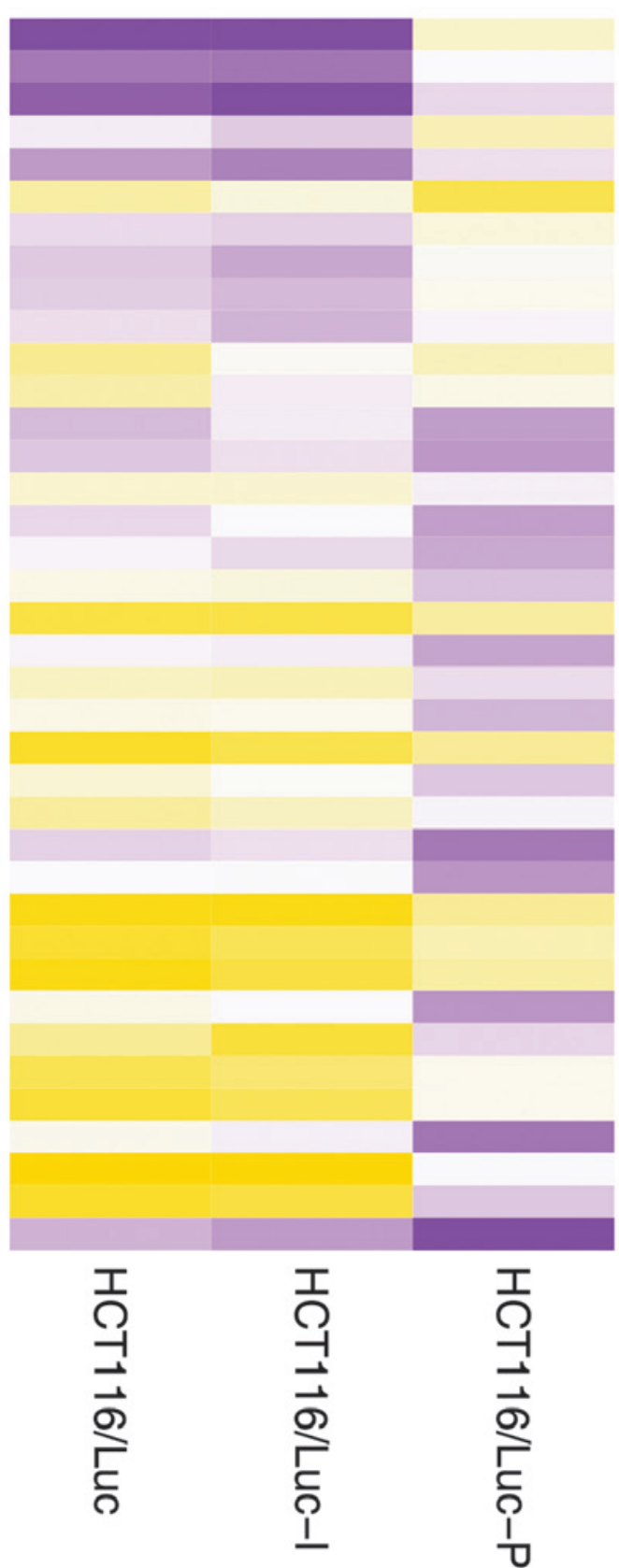

\begin{abstract}
hsa-miR-155-5p
hsa-miR-192-5p

hsa-miR-708-5p

hsa-miR-4281

hsa-miR-224-5p

hsa-miR-22-3p

hsa-miR-181a-5p

hsa-miR-3135b

hsa-miR-181b-5p

hsa-miR-30a-5p

hsa-miR-10b-5p

hsa-miR-10a-5p

hsa-miR-378a-3p

hsa-miR-378c

hsa-miR-2392

hsa-miR-378i

hsa-miR-151a-3p

hsa-miR-135a-5p

hsa-miR-221-3p

hsa-miR-301b

hsa-miR-135b-5p

hsa-miR-301a-3p

hsa-miR-141-3p

hsa-miR-151b

hsa-miR-151a-5p

hsa-miR-378b

hsa-miR-9-3p

hsa-miR-222-3p

hsa-miR-191-5p

hsa-miR-31-5p

hsa-miR-203a

hsa-miR-7-5p

hsa-miR-4324

hsa-miR-125b-5p

hsa-miR-145-5p

hsa-miR-100-5p

hsa-miR-99a-5p

hsa-miR-153
\end{abstract}

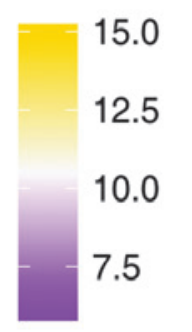

Figure 3. Heat map of the differentially expressed microRNAs. Stacking-hybridized universal tag assay was used for the high-throughput miRNA profiling and the miRNAs with differential expression are listed. 
expression level positively correlated with the grade of malignancy of the tumor (30). Hsa-miR-181b-5p was also revealed to be upreglulated in gastric cancer, which may correspond with lymph node invasion, nerve invasion and vascular invasion (31).

Further to the reported miRNAs, the function of other miRNAs in cancer require further investigation. The present profiling may be the first step toward delineating the differential expression of miRNAs in CRC cells located in colon and lung. This may enable researchers to elucidate the regulation associated with miRNAs in colorectal lung metastases. These miRNAs require further validation and functional analysis to evaluate whether they are important in the pathogenesis of colorectal lung metastases or are adopted as markers for the prediction of colorectal metastasis.

\section{Acknowledgements}

The authors would like to thank Assistant Investigator Kexiao Zheng and Professor Jiong Li (Suzhou Institute of Nano-tech and Nano-Bionics, Chinese Academy of Sciences) for providing the miRNA microarray assay support. The present study was funded by Medicine and Healthcare in Zhejiang Province General Studies Program (grant nos. 2012KYA027 and 2013KYA023).

\section{References}

1. Siegel R, Naishadham D and Jemal A: Cancer statistics. CA cancer J Clin 63: 11-30, 2013.

2. Cidón EU: The challenge of metastatic colorectal cancer. Clin Med Insights Oncol 4: 55-60, 2010.

3. Janjan NA, Crane C, Feig BW, et al: Improved overall survival among responders to preoperative chemoradiation for locally advanced rectal cancer. Am J Clin Oncol 24: 107-112, 2001.

4. Wang J,Rajput A,Kan JL, et al: Knockdown of Ron kinase inhibits mutant phosphatidylinositol 3-kinase and reduces metastasis in human colon carcinoma. J Biol Chem 284: 10912-10922, 2009.

5. Park JJ and Lee M: Increasing the $\alpha 2,6$ sialylation of glycoproteins may contribute to metastatic spread and therapeutic resistance in colorectal cancer. Gut Liver 7: 629-641, 2013.

6. Lee HJ, Lee M, Kang CM, et al: Identification of possible candidate biomarkers for local or whole body radiation exposure in C57BL/6 mice. Int J Radiat Oncol Biol Phys 69: 1272-1281, 2007.

7. Vassos N, Rau T, Merkel S, et al: Prognostic value of $\beta 1$ integrin expression in colorectal liver metastases. Int J Clin Exp Pathol 7 : 288-300, 2013

8. Zhang BH, Pan XP, Cobb GP and Anderson TA: microRNAs as oncogenes and tumor suppressors. Dev Biol 302: 1-12, 2007.

9. Zhang BH, Stellwag EJ and Pan XP: Large-scale genome analysis reveals unique features of microRNAs. Gene 443: 100-109, 2009.
10. Koturbash I, Zemp FJ, Pogribny I and Kovalchuk O: Small molecules with big effects: the role of the microRNAome in cancer and carcinogenesis. Mutat Res 722: 94-105, 2011.

11. Meng F, Henson R, Lang M, et al: Involvement of human micro-RNA in growth and response to chemotherapy in human cholangiocarcinoma cell lines. Gastroenterology 130: 2113-2129, 2006.

12. Xia L, Zhang D, Du R, et al: miR-15b and miR-16 modulate multidrug resistance by targeting BCL2 in human gastric cancer cells. Int J Cancer 123: 372-379, 2008

13. Khanna $\mathrm{C}$ and Hunter $\mathrm{K}$ : Modeling metastasis in vivo. Carcinogenesis 26: 513-523, 2005.

14. Duan D, Zheng KX, Shen Y, et al: Label-free high-throughput microRNA expression profiling from total RNA. Nucleic Acids Res 39: e154, 2011.

15. Zhang $\mathrm{X}$ and Li W: 5-Fluorouracil in combination with cisplatin alters the microRNA expression profile in the CNE nasopharyngeal carcinoma cell line. Mol Med Rep 6: 303-308, 2012.

16. Zhang BH, Stellwag EJ and Pan X: Large-scale genome analysis reveals unique features of microRNAs. Gene 443: 100-109, 2009

17. Yau TO, Wu CW, Dong Y, et al: microRNA-221 and microRNA-18a identification in stool as potential biomarkers for the non-invasive diagnosis of colorectal carcinoma. $\mathrm{Br} \mathrm{J}$ Cancer 111: 1765-1771, 2014

18. Saumet A, Mathelier A and Lecellier $\mathrm{CH}$ : The potential of microRNAs in personalized medicine against cancers. Biomed Res Int 2014: 642916, 2014.

19. Heneghan HM, Miller N and Kerin MJ: MiRNAs as biomarkers and therapeutic targets in cancer. Curr Opin Pharmacol 10: $543-550,2010$

20. Olson EN: MicroRNAs as therapeutic targets and biomarkers of cardiovascular disease. Sci Transl Med 6: 239ps3, 2014.

21. Chang GJ, Kaiser AM, Mills S, et al: Practice parameters for the management of colon cancer. Dis Colon Rectum 55: 831-843, 2012.

22. Portera CA Jr, Berman RS and Ellis LM: Molecular determinants of colon cancer metastasis. Surg Oncol 7: 183-195, 1998.

23. Yokota J: Tumor progression and metastasis. Carcinogenesis 21: 497-503, 2000.

24. Jemal A, Siegel R, Ward E, et al: Cancer statistics, 2007. CA Cancer J Clin 57: 43-66, 2007.

25. Gao W, Shen H, Liu L, et al: MiR-21 overexpression in human primary squamous cell lung carcinoma is associated with poor patient prognosis. J Cancer Res Clin Oncol 137: 557-566, 2011.

26. Jiang Y, Zhang M, He H, et al: MicroRNA/mRNA profiling and regulatory network of intracranial aneurysm. BMC Med Genomics 6: 36, 2013.

27. Sanchez-Diaz PC, Hsiao TH, Chang JC, et al: De-regulated microRNAs in pediatric cancer stem cells target pathways involved in cell proliferation, cell cycle and development. PLoS One 8: e61622, 2013.

28. Zawistowski JS, Nakamura K, Parker JS, et al: MicroRNA 9-3p targets $\beta 1$ integrin to sensitize claudin-low breast cancer cells to MEK inhibition. Mol Cell Biol 33: 2260-2274, 2013.

29. Giles KM, Brown RA, Epis MR, et al: miRNA-7-5p inhibits melanoma cell migration and invasion. Biochem Biophys Res Commun 430: 706-710, 2013

30. Wang K, Jia Z,Zou J, et al: Analysis of hsa-miR-30a-5p expression in human gliomas. Pathol Oncol Res 19: 405-411, 2013.

31. Chen G, Shen ZL, Wang L, et al: Hsa-miR-181a-5p expression and effects on cell proliferation in gastric cancer. Asian Pac J Cancer Prev 14: 3871-3875, 2013. 